\title{
SHORT PROOFS OF THREE THEOREMS ON HARMONIC FUNCTIONS
}

\author{
H. P. BOAS AND R. P. BOAS
}

(Communicated by Irwin Kra)

\begin{abstract}
We present elementary proofs-shorter than any others that we know-for three related theorems.
\end{abstract}

THEOREM I. A function $u$ that is harmonic and positive in the upper half-space $\left\{x \in \mathbf{R}^{n}: x_{n}>0\right\}$ and zero on the boundary hyperplane must be of the form $a x_{n}$.

When $n=2$, for instance, this implies that an entire function which maps the upper half-plane into itself and is real on the real axis is an affine function $a z+b$. Many proofs have been given, for instance [1-7]. Our proof is similar to the one given in [4] for the planar case.

THEOREM II. A function $u$ that is harmonic in $\mathbf{R}^{n}$ and bounded from one side by a polynomial must be a polynomial, and of no higher degree.

This is a strong form of Liouville's theorem.

THEOREM III. A function $f$, meromorphic in the whole complex plane, real on the real axis, with $\operatorname{Im} f(z) \geq 0$ when $\operatorname{Im} z \geq 0$, has the form

$$
f(z)=b_{0}+b_{1} z+\frac{c}{z}+\sum\left(\frac{A_{k}}{z-a_{k}}+\frac{A_{k}}{a_{k}}\right)
$$

where $b_{0} \in \mathbf{R}, b_{1} \geq 0, c \leq 0, a_{k} \in \mathbf{R} \backslash\{0\}, A_{k}<0$, and the series converges uniformly on every compact set that avoids the poles.

This is a theorem of Chebotarev and Meĭman [1, p. 197].

PROOF OF THEOREM I. By the reflection principle we may assume that $u$ is harmonic in the whole space $\mathbf{R}^{n}$, and that $u$ is an odd function of $x_{n}$. Write

$$
u(x)=\sum_{j=1}^{\infty} u_{j}(x)=\sum_{j=1}^{\infty} r^{j} u_{j}(\omega)
$$

where $x=r \omega, r=|x|$, and each $u_{j}$ is a homogeneous polynomial of degree $j$. The $u_{j}$ inherit from $u$ the properties of harmonicity and anti-symmetry in the variable $x_{n}$. In particular $u_{1}(x)=a x_{n}$, and the function $\left|u_{j}(x) / x_{n}\right|$ extends continuously to the unit sphere and has some upper bound $c_{j}$ there. Multiply (1) by $g(\omega)=$ $c_{k} \omega_{n}-u_{k}(\omega)$ and integrate over the unit sphere. Since spherical harmonics of

Received by the editors June 8, 1987.

1980 Mathematics Subject (Classification (1985 Revision). Primary 31B05, Secondary 30D30.

Key words and phrases. Positive harmonic functions, Liouville's theorem, meromorphic functions.

The first author partially supported by NSF grant number DMS- 8501758 . 
different degrees are orthogonal, and $u$ and $g$ have the same sign at each point, we obtain

$$
0 \leq a c_{k} r\left\|\omega_{n}\right\|^{2}-r^{k}\left\|u_{k}\right\|^{2}
$$

for each $k$. Since $r$ can be arbitrarily large, this implies that $u_{k}=0$ when $k>1$.

PROOF OF THEOREM II. Changing signs if necessary we have

$$
0 \leq p(r \omega)+\sum_{j=0}^{\infty} r^{j} u_{j}(\omega)
$$

where $p$ is a polynomial, say of degree $m$, and each $u_{j}$ is a homogeneous harmonic polynomial of degree $j$. Let $b_{j}$ be an upper bound for $\left|u_{j}(x)\right|$ on the unit sphere. Multiplying (2) by $b_{k}-u_{k}(\omega)$ and integrating over the unit sphere gives

$$
0 \leq O\left(r^{m}\right)-\int p(r \omega) u_{k}(\omega)-r^{k}\left\|u_{k}\right\|^{2}
$$

for each $k$. Since $u_{k}(\omega)$ is orthogonal to $p(r \omega)$ when $k>m$, the $u_{k}$ must vanish for such $k$. Hence $u$ is a polynomial of degree at most $m$.

Notice that this proof not only avoids using the Poisson formula, but also (when $n=2$ ) establishes a strong form of Liouville's theorem without appealing to Cauchy's theorem: namely, if $f$ is entire and $\lim \sup _{z \rightarrow \infty}|f(z)| /|z|^{m}<\infty$ then $f$ is a polynomial of degree at most $m$. Indeed if $m$ is even this condition implies that the real and imaginary parts of $f$ are bounded by a polynomial of degree $m$ in $x$ and $y$, and so the conclusion follows from Theorem II; if $m$ is odd the same argument applies to the function $z f(z)$.

PROOF OF THEOREM III. Since $f$ is real on the real axis, $f(\bar{z})=\overline{f(z)}$ and hence $\operatorname{Im} f(z) \leq 0$ when $\operatorname{Im} z \leq 0$. Since $f$ maps a neighborhood of a pole to a neighborhood of $\infty$, no neighborhood of a pole can lie entirely in either the upper or the lower half-plane: the poles must lie on the real axis. Near a pole $p$ the function $f$ behaves like $C(z-p)^{-m}$, and since the upper half of a neighborhood of $p$ maps into the upper half-plane, $m=1$ and $C<0$.

By composing $f$ with a real translation we may assume that 0 is not a pole. Let $a_{1}, a_{2}, \ldots$ be the poles of $f$ in increasing order of absolute value, and let $A_{k}$ be the (negative) residue of $f$ at $a_{k}$. In the annulus $\left\{z:\left|a_{n}\right|<|z|<\left|a_{n+1}\right|\right\}$ the function $f$ has a Laurent series $f(z)=\sum_{-\infty}^{\infty} \beta_{j} z^{j}$ with real coefficients $\beta_{j}$, and in particular

$$
\beta_{-1}=\sum_{k \leq n} A_{k}, \quad \beta_{1}=f^{\prime}(0)+\sum_{k \leq n} A_{k} / a_{k}^{2} .
$$

If $\left|a_{n}\right|<r<\left|a_{n+1}\right|$ then by hypothesis

$$
0 \leq \operatorname{Im} \int_{0}^{2 \pi} f\left(r e^{i \theta}\right) \sin \theta d \theta=\pi \beta_{1} r-\pi \beta_{-1} r^{-1},
$$

and so

$$
\sum_{k \leq n}\left|A_{k}\right|\left(a_{k}^{-2}-r^{-2}\right) \leq f^{\prime}(0) .
$$

Letting $r \rightarrow \infty$ we conclude that $\sum\left|A_{k}\right| / a_{k}^{2} \leq f^{\prime}(0)$.

The convergence of $\sum A_{k} / a_{k}^{2}$ implies that

$$
f(z)=g(z)+\sum\left(\frac{A_{k}}{z-a_{k}}+\frac{A_{k}}{a_{k}}\right)
$$


where the sum converges uniformly on compact subsets of $\mathbf{C} \backslash\left\{a_{k}\right\}$ and $g(z)=$ $\sum_{0}^{\infty} b_{j} z^{j}$ is entire (and $b_{j} \in \mathbf{R}$ ). Let $h_{n}(\theta)=n \sin \theta \pm \sin n \theta$. By expanding in binomial series we find for $r \neq\left|a_{k}\right|$ that

$$
\operatorname{Im} \int_{0}^{2 \pi} \frac{A_{k}}{r e^{i \theta}-a_{k}} h_{n}(\theta) d \theta \leq \pi(n+1) r\left|A_{k}\right| / a_{k}^{2} .
$$

Since $h_{n}(\theta)$ has the same sign as $\sin \theta$, we have by hypothesis

$$
0 \leq \pi^{-1} \operatorname{Im} \int_{0}^{2 \pi} f\left(r e^{i \theta}\right) h_{n}(\theta) d \theta \leq n b_{1} r \pm b_{n} r^{n}+(n+1) r \sum\left|A_{k}\right| / a_{k}^{2} .
$$

Therefore $b_{n}=0$ for $n \geq 2$. Setting $z=0$ shows that $b_{0}$ is real. Moreover $f^{\prime}(0)=b_{1}+\sum\left|A_{k}\right| / a_{k}^{2} \leq b_{1}+f^{\prime}(0)$, so $b_{1} \geq 0$. This completes the proof.

\section{REFERENCES}

1. N. G. Chebotarev and N. N. Melman, The Routh-Hurwitz problem for polynomials and entire functions, Trudy Mat. Inst. Steklov. 26 (1949). (Russian)

2. A. Dinghas, Über positive harmonische Funktionen in einem Halbraum, Math. Z. 46 (1940), 559-570.

3. Ü. Kuran, Harmonic majorizations in half-balls and half-spaces, Proc. London Math. Soc. 21 (1970), 614-636.

4. L. H. Loomis and D. V. Widder, The Poisson integral representation of functions which are positive and harmonic in a half-plane, Duke Math. J. 9 (1942), 643-645.

5. M. Tideman, Elementary proof of a uniqueness theorem for positive harmonic functions, Nordisk. Mat. Tidskrift 2 (1954), 95-96.

6. N. Tschebotareff, Über Realität von Nullstellen ganzer transzendenter Funktionen, Math. Ann. 99 (1928), 660-686.

7. M. Tsuji, On a positive harmonic function in a half-plane, Japan. J. Math. 15 (1939), 277-285.

Department of Mathematics, Texas A\&M University, College Station, TEXAS 77843

DEPARTMENT OF MATHEMATICS, NORTHWESTERN UNIVERSITY, EVANSTON, ILLINOIS, 60201 